\title{
DYNAMICAL MODELS FOR PRICES WITH DISTRIBUTED DELAYS
}

\author{
Gabriela MIRCEA 1 \\ Mihaela NEAMȚU 2 \\ Laura Mariana CISMAŞ ${ }^{3}$
}

DOI: $10.1515 /$ tjeb-2015-0010

\begin{abstract}
In the present paper we study some models for the price dynamics of a single commodity market. The quantities of supplied and demanded are regarded as a function of time. Nonlinearities in both supply and demand functions are considered. The inventory and the level of inventory are taken into consideration. Due to the fact that the consumer behavior affects commodity demand, and the behavior is influenced not only by the instantaneous price, but also by the weighted past prices, the distributed time delay is introduced. The following kernels are taken into consideration: demand price weak kernel and demand price Dirac kernel. Only one positive equilibrium point is found and its stability analysis is presented. When the demand price kernel is weak, under some conditions of the parameters, the equilibrium point is locally asymptotically stable. When the demand price kernel is Dirac, the existence of the local oscillations is investigated. A change in local stability of the equilibrium point, from stable to unstable, implies a Hopf bifurcation. A family of periodic orbits bifurcates from the positive equilibrium point when the time delay passes through a critical value. The last part contains some numerical simulations to illustrate the effectiveness of our results and conclusions.
\end{abstract}

Keywords: $\quad$ delay differential equations, delayed economic models, local stability, price dynamics, Hopf bifurcation.

\section{JEL Classification: C61, C62, D24, D50.}

\footnotetext{
${ }^{1}$ Assistant Professor PhD, West University of Timişoara, Romania

${ }^{2,3}$ Professor PhD, West University of Timişoara, Romania
} 


\section{Introduction}

Trade cycles, business cycle and fluctuations in the price and supply of various commodities have attracted the attention of economists for well over on hundred years and possible more the thousands of years [11].

Huang et al. [4] establish a class of economic models with two delays, where the delay is considered in both the production process and consumer consumption. The dynamics of this system have been investigated. In [1], Belair and Mackey develop a model for the dynamics of price adjustment in a single commodity market. Zhai et al. [10] modify and further develop the Rayleigh price model. They can more accurately illustrate the economic phenomena with price varying.

Based on [4], in this paper we analyze economic models which describe the dynamics of the prices for a single commodity market. The market price $p(t)$ is described with the function $D(\cdot)$ and $S(\cdot)$, respectively, denote the demand and supply schedules are given by $p_{1}(t)$ (demand price) and $p_{2}(t)$ (supply price), respectively. We consider that consumer behavior is affected by the information regarding past price. Thus, the demand for a commodity is a weighted function $p_{1}(t)$ of past prices. It is described by the function $k_{1}(t)$ called demand price kernel. The relation between current market price $p(t)$ and the supply price is given by the supply price kernel $k_{2}(t)$. These type of kernels are introduced in [1]. Also, we consider the demand and supply functions given in [4].

The paper is structured as follows. In section 2, the economic models with demand and supply price kernels are presented. In section 3, we consider the coefficients of the kernels as parameters and using the Hopf bifurcation theory we discuss the stability of the equilibrium point for the models from section 2 . The following kernels are taken into consideration: demand price weak kernel, supply weak kernel, demand price Dirac kernel, supply price Dirac kernel. In section 4 numerical simulations are given to illustrate the obtained results. Section 5 gives some conclusions and future researches.

\section{Economic Models with Distributed Delays}

Considering a single commodity market, the quantity of supplied and demand can be regarded as the function of time, namely $G_{0}(t)$ and $D_{0}(t)$. The inventory and the level of inventory are recorded, respectively, as $S(t)$ and $S_{0}$.

\section{DE GRUYTER OPEN}

Timisoara Journal of Economics and Business | ISSN: 2286-0991 | www.tjeb.ro Year 2015 | Volume 8 | Issue 1s | Pages: 91-102 
Let $p(t)$ be the price at time t, so that the rate of price increases in proportion to the difference between $S_{0}$ and $S(t)[4]$ :

$$
\dot{p}(t)=-a\left(S(t)-S_{0}\right), a>0
$$

where $a$ is a positive real number depending on the speed of price adjustment, recording $S(t)$ as:

$$
S(t)=S(0)+\int_{0}^{t}\left(G_{0}(s)-D_{0}(s)\right) d s
$$

In the traditional cobweb model, demand function is a function of price. In [4], the demand function is:

$$
D_{0}(t)=a_{1}-a_{2} p(t)-f(p(t)) \dot{p}(t)
$$

where $a_{1}>0, a_{2}>0, a_{2}$ represents the sensitive degree of consumers to the increase of commodity price; $f(p(t))$ is the level of the price relying on the rate of increase and $f: R_{+} \rightarrow R_{+}$is a derivative function with $f^{\prime}(x)>0, x \in R_{+}$.

In generally, the supply function is monotone increasing about price, but it is considered that as price goes up, the supply could not unlimitedly increase, thus the supply function is [4]:

$$
G_{0}(t)=b_{1}+b_{2} g(p(t))
$$

where $g: R_{+} \rightarrow R_{+}$is given by:

$$
g(x)=\frac{x}{b_{3}+x}
$$

and $b_{1}>0, b_{2}>0, b_{3}>0$.

From (1), (2) with (3) and (4), we have:

$$
\ddot{p}(t)=-a\left(a_{2} p(t)+f(p(t)) \dot{p}(t)+b_{2} g(p(t))+b_{1}-a_{1}\right)
$$

In specifying how consumer behavior affects commodity demand, we assume that this behavior is governed by an integration of information regarding past price. Thus, demand for a commodity is a weighted function $p_{1}(t)$ of past prices. In [1], the price $p_{1}(t)$ is called demand price and it is defined by:

\section{DE GRUYTER OPEN}


Mircea, G., Neamțu, M. \& Cismaş, L. M. (2015).

Dynamical Models for Prices with Distributed Delays

$$
p_{1}(t)=\int_{-\infty}^{0} k_{1}(t-s) p(s) d s
$$

where $k_{1}$ is called the demand price kernel.

The function $k_{1}:[0,+\infty) \rightarrow[0,+\infty)$ is pricewise continuous and it verifies ([6]):

$$
\int_{0}^{\infty} k_{1}(s) d s=1, \quad \int_{0}^{\infty} s k_{1}(s) d s<\infty
$$

In [1], the price $p_{2}(t)$ is called supply price and is defined by:

$$
p_{2}(t)=\int_{-\infty}^{0} k_{2}(t-s) p(s) d s
$$

where $k_{2}$ is called the supply price kernel. The function $k_{2}:[0,+\infty) \rightarrow[0,+\infty), k_{2}$ is pricewise continuous and it verifies ([6]):

$$
\int_{0}^{\infty} k_{2}(s) d s=1, \quad \int_{0}^{\infty} s k_{2}(s) d s<\infty .
$$

The demand function with demand price $p_{1}(t)$ is given by:

$$
D_{1}(t)=a_{1}-a_{2} p(t)-f(p(t)) \dot{p}(t)
$$

and the supply function with supply price $p_{2}(t)$ is given by:

$$
G_{2}(t)=b_{1}+b_{2} g(p(t))
$$

Using the function $D_{0}(t), D_{1}(t), G_{0}(t), G_{2}(t)$ we obtain the models:

$$
\dot{p}(t)=a\left(S_{0}-S_{01}(t)\right)
$$

where

$$
\begin{gathered}
S_{01}(t)=S_{01}(0)+\int_{0}^{t}\left(G_{0}(s)-D_{1}(s)\right) d s, \\
\dot{p}(t)=a\left(S_{0}-S_{20}(t)\right)
\end{gathered}
$$

DE GRUYTER OPEN
Timisoara Journal of Economics and Business | ISSN: 2286-0991 | www.tjeb.ro Year 2015 | Volume 8 | Issue 1s | Pages: 91-102 
Mircea, G., Neamțu, M. \& Cismaş, L. M. (2015).

Dynamical Models for Prices with Distributed Delays

where

$$
\begin{gathered}
S_{20}(t)=S_{20}(0)+\int_{0}^{t}\left(G_{2}(s)-D_{0}(s)\right) d s, \\
\dot{p}(t)=a\left(S_{0}-S_{21}(t)\right)
\end{gathered}
$$

where

$$
S_{21}(t)=S_{21}(0)+\int_{0}^{t}\left(G_{2}(s)-D_{1}(s)\right) d s
$$

Using (3), (4), (11), (12), the models (13), (15) and (17) are given by the following equations:

$$
\begin{aligned}
& \ddot{p}(t)=-a\left(a_{2} p_{1}(t)+f(p(t)) \dot{p}(t)+b_{2} g(p(t))+b_{1}-a_{1}\right) \\
& \ddot{p}(t)=-a\left(a_{2} p(t)+f(p(t)) \dot{p}(t)+b_{2} g\left(p_{2}(t)\right)+b_{1}-a_{1}\right) \\
& \ddot{p}(t)=-a\left(a_{2} p_{1}(t)+f(p(t)) \dot{p}(t)+b_{2} g\left(p_{2}(t)\right)+b_{1}-a_{1}\right)
\end{aligned}
$$

In what follows, we analyze the equation (6) and (19). They can be rewritten as:

$$
\begin{aligned}
& \dot{p}(t)=q(t) \\
& \dot{q}(t)=-a f(p(t)) q(t)-a a_{2} p(t)-a b_{2} g(p(t))+a\left(a_{1}-b_{1}\right) \\
& \dot{p}(t)=q(t) \\
& \dot{q}(t)=-a f(p(t)) q(t)-a a_{2} p_{1}(t)-a b_{2} g(p(t))+a\left(a_{1}-b_{1}\right)
\end{aligned}
$$

We analyze the models (22) and (23), for the following kernels:

1. $k_{1}(s)=d_{1} e^{-d_{1} s}, d_{1}>0$, the demand price weak kernel;

2. $k_{1}(s)=\delta\left(s-\tau_{1}\right), \tau_{1}>0$, the demand price Dirac kernel. 


\section{Stability Analysis and the Existence of the Hopf Bifurcation}

At first, we will show that the system (22) and (23) have only one positive equilibrium point under some assumptions.

Proposition 1. If $a_{1}>b_{1}$, then the systems (22) and (23) have only one positive equilibrium point $\left(p^{*}, 0\right)$, where $p^{*}$ is the positive solution of the equation:

$$
a_{2} p^{2}+\left(a_{2} b_{3}+b_{2}+b_{1}-a_{1}\right) p+b_{3}\left(b_{1}-a_{1}\right)=0 .
$$

The proof is obtained by vanishing the right parts of the above equations.

The analysis of the local stability of the system (22) and (23) can be done analyzing the characteristic equation of the linearized system in $\left(p^{*}, 0\right)$.

\subsection{Stability analysis of system (22)}

With the help of the coordinate translation:

$$
x(t)=p(t)-p^{*}, y(t)=q(t)
$$

system (22) can be rewritten in the following form:

$$
\begin{aligned}
& \dot{x}(t)=y(t) \\
& \left.\dot{y}(t)=-a f\left(x(t)+p^{*}\right) y(t)-a a_{2}(x(t))+p^{*}\right)-a b_{2} g\left(x(t)+p^{*}\right)+a\left(a_{1}-b_{1}\right) .
\end{aligned}
$$

The linearized system $(26)$ at $(0,0)$ is:

$$
\begin{aligned}
& \dot{u}_{1}(t)=u_{2}(t) \\
& u_{2}(t)=-\left(a a_{2}+a b_{2} b_{3} \beta_{2}\right) u_{1}(t)-a \beta_{1} u_{2}(t),
\end{aligned}
$$

where $\beta_{1}=f\left(p^{*}\right), \beta_{2}=\frac{1}{\left(p_{3}+p^{*}\right)^{2}}$.

Proposition 2. If $a_{1}>b_{1}$ the equilibrium point $\left(p^{*}, 0\right)$ is locally asymptotically stable.

Proof. The characteristic equation (22) is given by:

$$
\lambda^{2}+a \beta_{1} \lambda+a a_{2}+a b_{2} b_{3} \beta_{2}=0
$$

\section{DE GRUYTER OPEN}


Because $a \beta_{1}>0$ and $a\left(a_{2}+b_{2} b_{3} \beta_{2}\right)>0$, then all roots of (28) have negative real part. In this case the equilibrium point $\left(p^{*}, 0\right)$ is locally asymptotically stable.

\subsection{Stability analysis of system (23)}

With the help of the coordinate translation (25) system (23) is given by:

$$
\begin{aligned}
& \dot{x}(t)=y(t) \\
& \dot{y}(t)=-a f\left(x(t)+p^{*}\right) y(t)-a a_{2}\left(x_{1}(t)+p^{*}\right)-a b_{2} g\left(x(t)+p^{*}\right)+a\left(a_{1}-b_{1}\right)
\end{aligned}
$$

where

$$
x_{1}(t)=\int_{-\infty}^{0} k_{1}(t-s) x(s) d s
$$

The linearized system $(29)$ at $(0,0)$ is:

$$
\begin{aligned}
& \dot{u}_{1}(t)=u_{2}(t) \\
& u_{2}(t)=-a b_{2} b_{3} \beta_{2} u_{1}(t)-a a_{2} \int_{-\infty}^{0} k_{1}(t-s) u_{1}(s) d s-a \beta_{1} u_{2}(t)
\end{aligned}
$$

where $\beta_{1}=f\left(p^{*}\right), \beta_{2}=\frac{1}{\left(b_{3}+p^{*}\right)^{2}}$.

The characteristic equation of system (31) is given by:

$$
\lambda^{2}+a \beta_{1} \lambda+a b_{2} b_{3} \beta_{2}+a a_{2} \int_{-\infty}^{0} k_{1}(-s) e^{-\lambda s} d s=0
$$

Proposition 3. If $a_{1}>b_{1}$ and $k_{1}$ is the demand price weak kernel, the equilibrium point $\left(p^{*}, 0\right)$ is locally asymptotically stable for all $d_{1}>0$.

Proof. If $k_{1}(s)=d_{1} e^{-d_{1} s}$ then $\int_{-\infty}^{0} k_{1}(t-s) e^{-\lambda s} d s=\frac{d_{1}}{\lambda+d_{1}}$. Eq. (32) becomes:

$$
\lambda^{3}+m_{2} \lambda^{2}+m_{1} \lambda+m_{0}=0
$$

DE GRUYTER OPEN 
Mircea, G., Neamțu, M. \& Cismaş, L. M. (2015).

Dynamical Models for Prices with Distributed Delays

where

$$
m_{2}=d_{1}+a \beta_{1}, m_{1}=a\left(\beta_{1}+b_{2} b_{3} \beta_{2}+a_{2}\right), m_{0}=a b_{2} b_{3} d_{1} \beta_{2} .
$$

From (34) we have $m_{i}>0, i=0,1,2$.

Let $\psi_{1}:[0, \infty) \rightarrow R$ be a continuously differentiable function defined by:

$$
\psi_{1}\left(d_{1}\right)=m_{1} m_{2}-m_{0}
$$

From (34) and (35) we have:

$$
\psi_{1}\left(d_{1}\right)=a\left(\left(d_{1}+a \beta\right)\left(\beta_{1}+a_{2}\right)+a \beta_{1} \beta_{2} b_{2} b_{3}\right)
$$

From (36), we have $\psi_{1}\left(d_{1}\right)>0$ for $d_{1}>0$. The Routh-Hurwicz criterion implies that all roots of eq. (33) have negative real part for all $d_{1}>0$. Then, the equilibrium point $\left(p^{*}, 0\right)$ is locally asymptotically stable.

Now, let $k_{1}$ be the demand price Dirac kernel. The characteristic equation (31) is given by:

$$
\lambda^{2}+a \beta_{1} \lambda+a b_{2} b_{3} \beta_{2}+a a_{2} e^{-\lambda \tau_{1}}=0
$$

Proposition 4. If $a_{1}>b_{1}, a_{2}<b_{2} b_{3} \beta_{2}, a \beta_{1}^{2}-2 b_{2} b_{3} \beta_{2}<0$ and $\tau_{1}=\tau_{11}$, then eq. (37) has purely imaginary roots $\lambda= \pm i \omega_{11}$ when:

$$
\tau_{11}=\frac{1}{\omega_{11}}\left(\arccos \left(\frac{\omega_{11}^{2}-a_{2} b_{2} b_{3} \beta_{2}}{a a_{2}}\right)+2 n \pi\right), n=0,1,2, \ldots
$$

and $\omega_{11}$ is a positive real root of the equation:

$$
\omega^{4}+a\left(a \beta_{1}^{2}-2 b_{2} b_{3} \beta_{2}\right) \omega^{2}+a^{2}\left(b_{2}^{2} b_{3}^{2} \beta_{2}^{2}-a_{2}^{2}\right)=0
$$

Proof. For $\tau_{1}=0,(37)$ is (28). Eq. (28) has roots with negative real part. If $\lambda=i \omega_{11}$ is a root of (37) then:

DE GRUYTER OPEN
Timisoara Journal of Economics and Business | ISSN: 2286-0991 | www.tjeb.ro

Year 2015 | Volume 8 | Issue 1s | Pages: 91-102 
Mircea, G., Neamțu, M. \& Cismaş, L. M. (2015).

Dynamical Models for Prices with Distributed Delays

$$
a a_{2} \cos \left(\omega \tau_{1}\right)=\omega^{2}-a b_{2} b_{3} \beta_{2}, \quad \text { a } a_{2} \sin \left(\omega \tau_{1}\right)=a \beta_{1} \omega
$$

From (40) we obtain eq. (39). Because $b_{2} b_{3} \beta_{2}-a_{2}>0$ and $a \beta_{1}^{2}-2 b_{2} b_{3} \beta_{2}<0$ then eq. (39) has positive roots. From (40), for the positive root $\omega_{11}$, we obtain (38).

Proposition 5. For $\tau_{11}$ and $\omega_{11}$, given by (38) and (39), we have:

$$
\left.\frac{d \lambda\left(\tau_{1}\right)}{d \tau_{1}}\right|_{\tau_{1}=\tau_{11}, \lambda=i \omega_{11}}>0
$$

Proof. Taking the derivative of eq. (37) with respect to ${ }^{\tau_{1}}$, we have:

$$
\left(\frac{d \lambda}{d \tau_{1}}\right)^{-1}=\frac{\left(2 \lambda+a \beta_{1}\right) e^{\lambda \tau_{2}}}{a a_{2} \lambda}-\frac{\tau_{1}}{\lambda}
$$

From (42) we have:

$$
\operatorname{Re}\left(\frac{d \lambda}{d \tau_{1}}\right)_{\tau_{1}=\tau_{11}, \lambda=i \omega_{11}}^{-1}=\frac{1}{a^{2} a_{2}^{2}}\left[2 \omega_{11}^{2}-2 a b_{2} b_{3} \beta+a^{2} \beta_{1}^{2}\right]>0
$$

Based on Proposition 4 and Proposition 5 and the classical Hopf bifurcation, we have:

Proposition 6 ([2], [3], [5]) Assume the conditions from Proposition 4 hold. Then, we have the following results:

1. If $\tau_{1} \in\left[0, \tau_{11}\right)$ all roots of (37) have negative real part. Namely the equilibrium point $\left(p^{*}, 0\right)$ of system (23) is locally asymptotically stable.

2. If $\tau_{1}=\tau_{11}$ eq. (37) has a pair of purely imaginary roots $\pm i \omega_{11}$, all the other roots have negative real part. That is to say, system (23) undergoes a Hopf bifurcation at $\tau_{1}=\tau_{11}$.

3. If $\tau_{1}>\tau_{11}$ eq. (37) has roots have positive real part. The equilibrium point $\left(p^{*}, 0\right)$ of (23) is unstable. 
Mircea, G., Neamțu, M. \& Cismaş, L. M. (2015). of Economics and Business

Dynamical Models for Prices with Distributed Delays

\section{Numerical Simulation}

For the numerical simulation we consider the following parameters: $a_{1}=125, a_{2}=2$, $a=0.08, b_{1}=85, b_{2}=180, b_{3}=12$. The equilibrium point is $(2.82921,0)$.

For system (22), the equilibrium point is locally asymptotically stable:

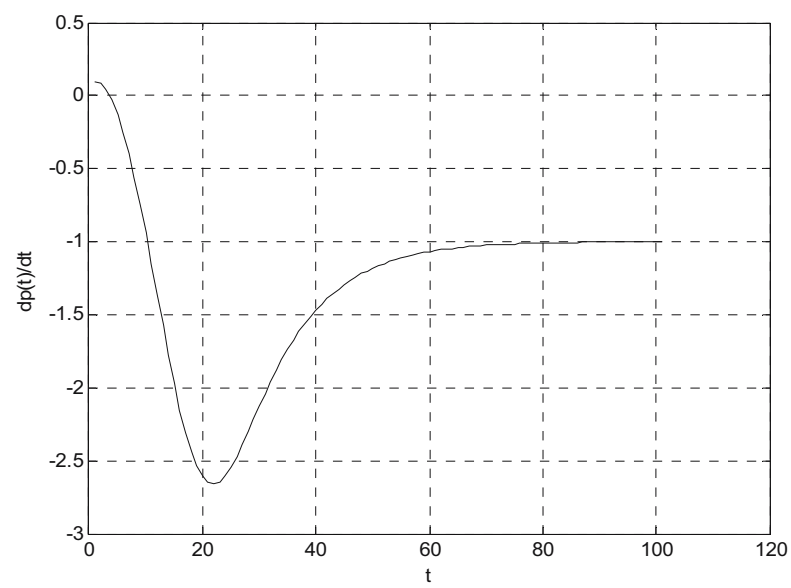

Figure 1. $(t, \dot{p}(t))$

For system (23), $d_{1}=0.5, k_{1}(s)=d_{1} e^{-d_{1} s}$, the equilibrium point is locally asymptotically stable:

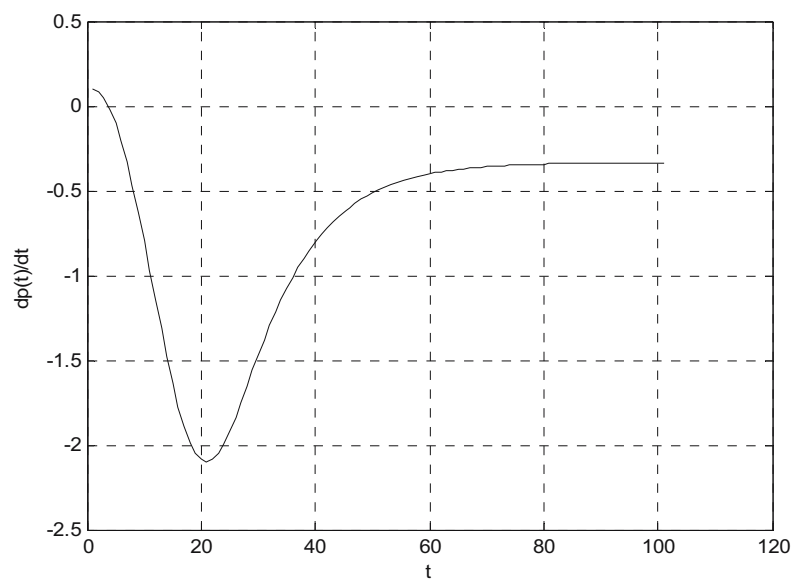

Figure 2. $(t, \dot{p}(t))$

DE GRUYTER OPEN
Timisoara Journal of Economics and Business | ISSN: 2286-0991 | www.tjeb.ro Year 2015 | Volume 8 | Issue 1s | Pages: 91-102 
Mircea, G., Neamțu, M. \& Cismaş, L. M. (2015).

Dynamical Models for Prices with Distributed Delays

For system (23), when $k_{1}$ is the demand price Dirac kernel, then for $\tau_{11}=0.7$ there exists a Hopf bifurcation. For $\tau_{1} \in\left[0, \tau_{11}\right)$, the equilibrium point of the system (23) is locally asymptotically stable. For $\tau_{1}>\tau_{11}$, it is unstable.

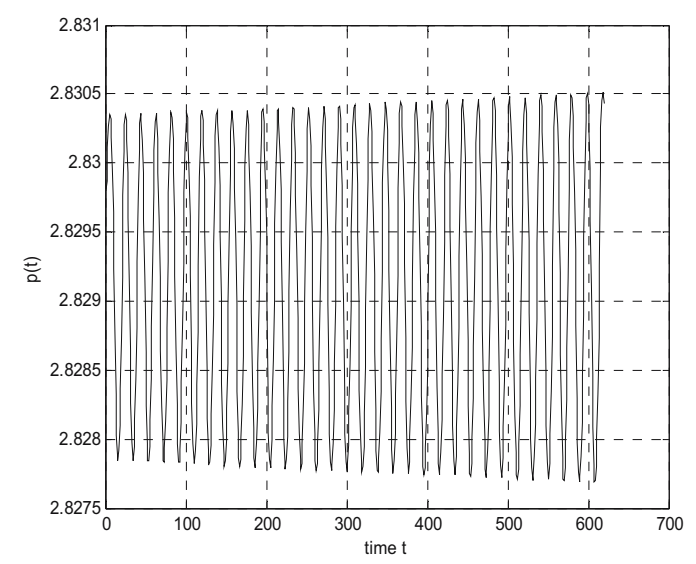

Figure 3. $(t, p(t)), \tau_{11}=0.7$

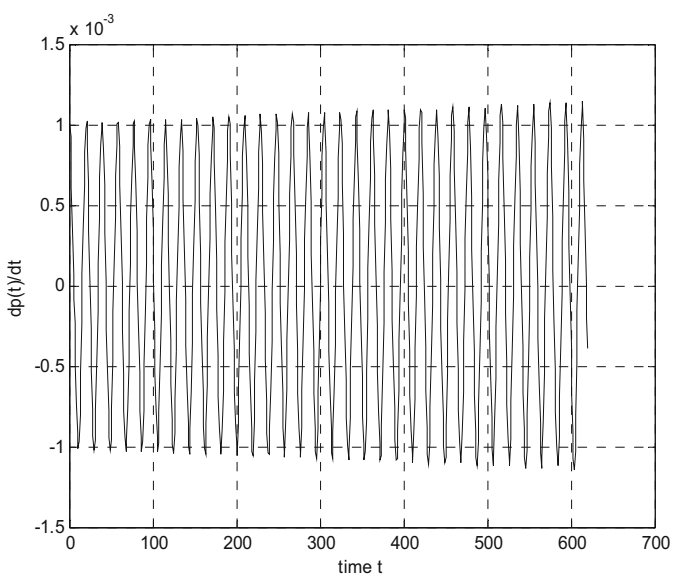

Figure 4. $(t, \dot{p}(t)), \tau_{11}=0.7$

\section{Conclusions}

The demand price kernel and supply price kernel in both production process and consumer consumption have been considered. Two types of kernels have been taken into account: weak and Dirac. For the developed models, the dynamics has been investigated. The equilibrium point has determined and the existence of the period solutions has been studied. For the numerical simulations we have used Maple and Matlab and the obtained figures verify the theoretical findings.

For the uniform distribution and the strong kernel, a similar analysis will be carried out in the next paper. As in [7], [8], [9] the stochastic approach will be taken into consideration.

\section{Acknowledgements:}

We would like to offer special thanks to Dumitru Opris, professor at West University of Timisoara for the numerous discussions on the topic of the paper.

DE GRUYTER OPEN
Timisoara Journal of Economics and Business | ISSN: 2286-0991 | www.tjeb.ro

Year 2015 | Volume 8 | Issue 1s | Pages: 91-102 
Mircea, G., Neamțu, M. \& Cismaş, L. M. (2015).

Dynamical Models for Prices with Distributed Delays

\section{References}

Belair, J. \& Mackey, M.C. (1989). Consummer memory and price fluctuations in commodity markers: An integrodifferential model. Journal of dynamics and differential equation, 1(3), 299-325.

Hale, J.K. \& Lunel, S. V. (1993). Introduction to functional differential equations. Springer Verlag, New York.

Hasssard, B.D., Kazarinoff, N.D. \& Wan Y.H. (1981). Theory and applications of Hopf bifurcation, vol. 41 of London Mathematical Society. Lecture Notes Series, Cambridge University Press, Cambridge, UK.

Huang, C., Peng, C., Chen, X. \& Wen, F. (2013). Dynamics Analysis of a Class of Delayed Economic Model. Abstract and Applied Analysis, 2013, Article ID962738, http://dx.doi.org/10.1155/2013/962738

Kutznenetsov, Y.A. (1995). Elements of applied bifurcation theory, Springer Verlag, New York

Li, X., Ruin, S. \& Wei, J. (1999). Stability and bifurcation in delay-differential equation with two delays. Journal of Math. Analysis and Applications, 236(2), 254-280.

Mircea, G., Neamtu, M. \& Opris, D. (2011). Uncertain, stochastic and fractional dynamic systems with delay. Applications, Lambert Academic Publishing, Germany

Mircea, G., Neamtu, M., Bundau, O. \& Opris, D. (2012). Uncertain and stochastic financial models with multiple delays. International journal of bifurcation and chaos, 22(6), 1250131-1-19, DOI: 10.1142/s0218127412501313

Sirghi, N. \& Neamtu, M. (2013). Deterministic and stochastic advertising diffusion model with delay. WSEAS Transaction on system and control, 4(8), 141-150.

Zhai, Y., Bai, H., Xiong, Y., \& Ma X. (2013). Hopf bifurcation analysis for the modified Rayleight price model with time delay. Abstract and applied analysis, volume 2013, article ID 290497, http://dx.doi.org/10.1155/2013/290497

Weidenbaum, M.L. \& Vogt, S.C. (1988). Are economic forecast any good? Mathematical and Computing Modelling, 11, 1-5.

DE GRUYTER OPEN 